${ }^{1}$ Centro de Enseñanza y Aprendizaje, Facultad de Medicina, Universidad de Chile. Santiago, Chile. ${ }^{2}$ Departamento de Educación en Ciencias de la Salud, Facultad de Medicina, Universidad de Chile. Santiago, Chile. anterno de Medicina. bLicenciado en Filosofía. Doctor en Psicología y Educación.

Fuente de apoyo financiero: El estudio fue financiado con recursos departamentales. Los autores declaran no tener conflictos de interés.

Recibido el 11 de noviembre de 2020, aceptado el 23 de marzo de 2021.

Correspondencia a Nicolás Ortiz-López Av. Independencia 1027, Independencia, Región Metropolitana. nicolas.ortiz@ug.uchile.cl

\section{Las experiencias de los estudiantes de medicina LGBTQ+ durante su formación: Una revisión sistemática}

\author{
NICOLÁS ORTIZ-LÓPEZ ${ }^{1, \mathrm{a}}$, OSCAR JEREZ YÁÑEZ, \\ FELIPE CORTÉS-CHAU ${ }^{1, a}$, JONATHAN RODRÍGUEZ-CABELLO ${ }^{1, a}$
}

\section{Experiences of medical students belonging to sexual and gender diversity: a systematic review}

Background: Students belonging to sexual and gender diversity experience chronic stress due to stigmatization and discrimination. Aim: To identify the experiences of lesbian, gay, bisexual, transgender/transsexual, and queer (LGB$T Q+)$ medical students. Material and Methods: Systematic literature review using the PRISMA protocol in PubMed, ERIC, EMBASE, and LILACS databases. Articles published in Spanish or English were considered. Three authors independently reviewed and synthesized information from the selected articles, according to the PRISMA criteria. Results: Fifteen studies met the inclusion criteria. Forty-three experiences were reported, which were finally classified into four categories: i) Relationship between peers in the educational context (23\%), ii) Relationship between students and teachers in the educational context (23\%), iii) Relationship with the educational institution (34\%), and iv) Curriculum and training experience (19\%). The relationship with the educational institution was identified as the most relevant category. Students with a strong sense of belonging to their institution were more likely to be persistent and make an effort in learning. The second most relevant experiences, mainly negative, derive from interactions with peers and teachers. Conclusions: LGBTQ+ medical students still experience more discrimination than inclusion during their training. Therefore, medical schools should render medical education a more inclusive space for the LGBTQ+ population.

(Rev Med Chile 2021; 149: 1058-1069)

Key words: Education, Medical; Schools, Medical; Sexual and Gender Minorities; Students, Medical; Systematic Review.
$\mathrm{E}$ s importante que el entorno de aprendizaje sea inclusivo para aquellos estudiantes que pertenecen a minorías, no solo para garantizar que los miembros de dichos grupos tengan éxito, sino también para modelar un ambiente de respeto e inclusión hacia todos miembros de la sociedad ${ }^{1}$. En efecto, uno de los objetivos de la educación médica es garantizar el desarrollo de competencia cultural en la formación de profesio- nales, para que puedan brindar atención médica a una amplia variedad de poblaciones ${ }^{2,3}$.

Particularmente, los estudiantes pertenecientes a la diversidad sexual y de género (DSG), es decir, la población lesbiana, gay, bisexual, transgénero/ transexual y queer (LGBTQ+), experimentan estrés crónico debido a la estigmatización y la discriminación, lo que debilita su capacidad de centrarse en lo académico ${ }^{4}$. Asimismo, en la educa- 
ción superior existe cierta resistencia a la inclusión de la población LGBTQ+, la cual crea un clima que inhibe a muchas personas de ser abiertas sobre su orientación sexual o identidad de género ${ }^{5}$. Este clima perpetúa que aquellos estudiantes continúen sintiéndose vulnerables y marginados ${ }^{5,6}$.

El objetivo de este estudio es brindar respuesta a la pregunta: ¿Cuáles son las experiencias de discriminación o inclusión de los estudiantes de medicina LGBTQ+?

\section{Método}

\section{Protocolo de revisión}

Esta revisión sistemática se realizó siguiendo las directrices del protocolo Preferred Reporting Items for Systematic Reviews and Meta-Analyses $\left(\right.$ PRISMA) ${ }^{7}$ (Figura 1).

\section{Estrategia de búsqueda}

Se realizó una búsqueda en cuatro bases de datos para artículos publicados en revistas revisadas por pares. No se aplicaron filtros por fecha o idioma. Se utilizó vocabulario controlado (Tabla 1).
Además, se realizaron búsquedas manuales en las referencias de los artículos seleccionados.

\section{Tamizaje de relevancia y criterios de inclusión}

Los títulos y resúmenes de los artículos se examinaron utilizando un formulario diseñado a priori que consta de dos preguntas: si la cita describió la investigación primaria sobre estudiantes de medicina de las DSG y si era relevante para la pregunta de investigación. Se incluyeron estudios originales, cualitativos y cuantitativos, que describen las experiencias de estudiantes de medicina pertenecientes a la DSG. Se excluyeron artículos centrados en estudiantes no médicos o en pacientes LGBTQ+, revisiones, editoriales y comentarios.

\section{Proceso de selección}

Una vez eliminados los artículos duplicados, tres autores revisaron de forma independiente títulos y resúmenes de cada estudio identificado en la búsqueda. Se seleccionaron aquellos que cumplían con los criterios de inclusión y las discrepancias fueron resueltas por un cuarto autor, de los cuales se revisó el texto completo.

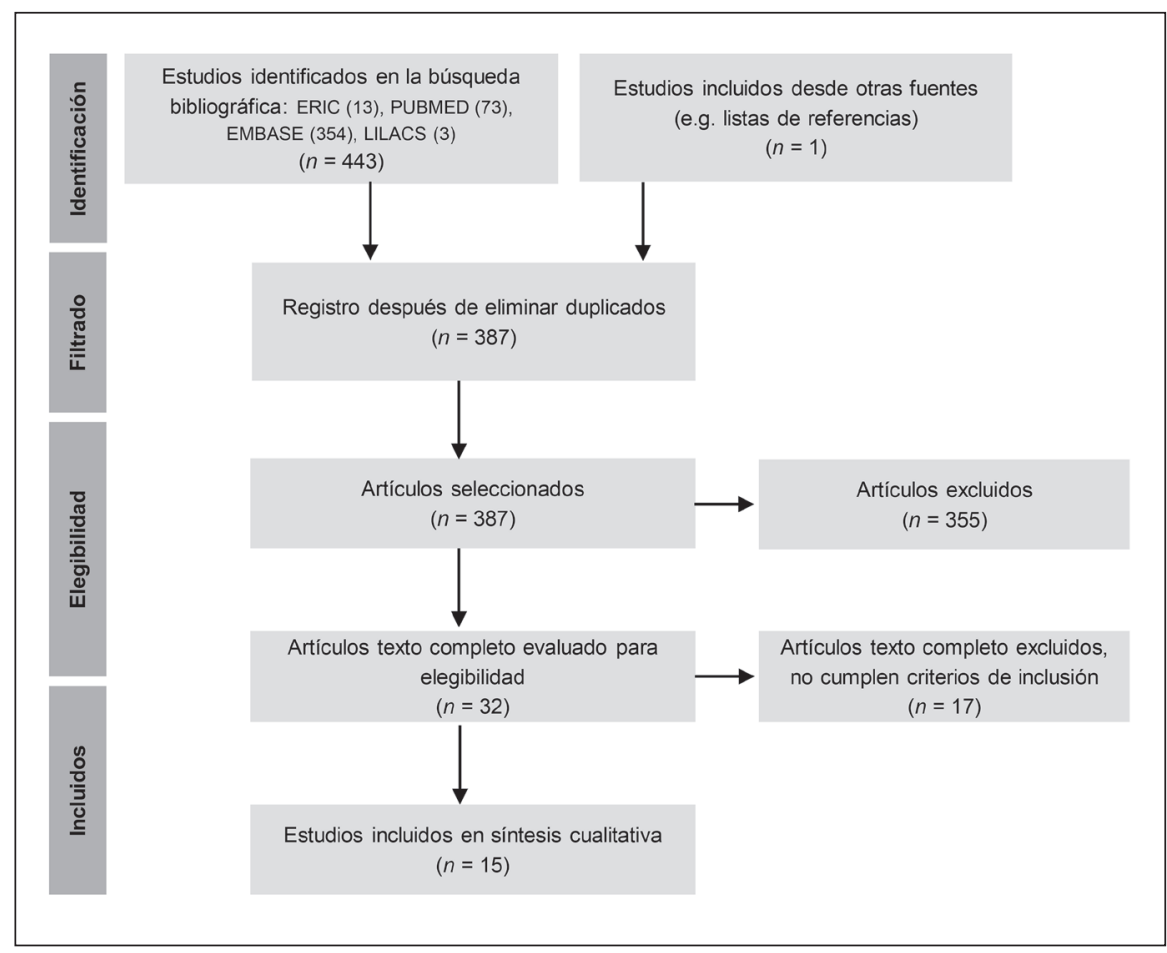

Figura 1. Flujograma de búsqueda de acuerdo a los criterios PRISMA. 
Tabla 1. Identificación y expansión de los conceptos base de búsqueda

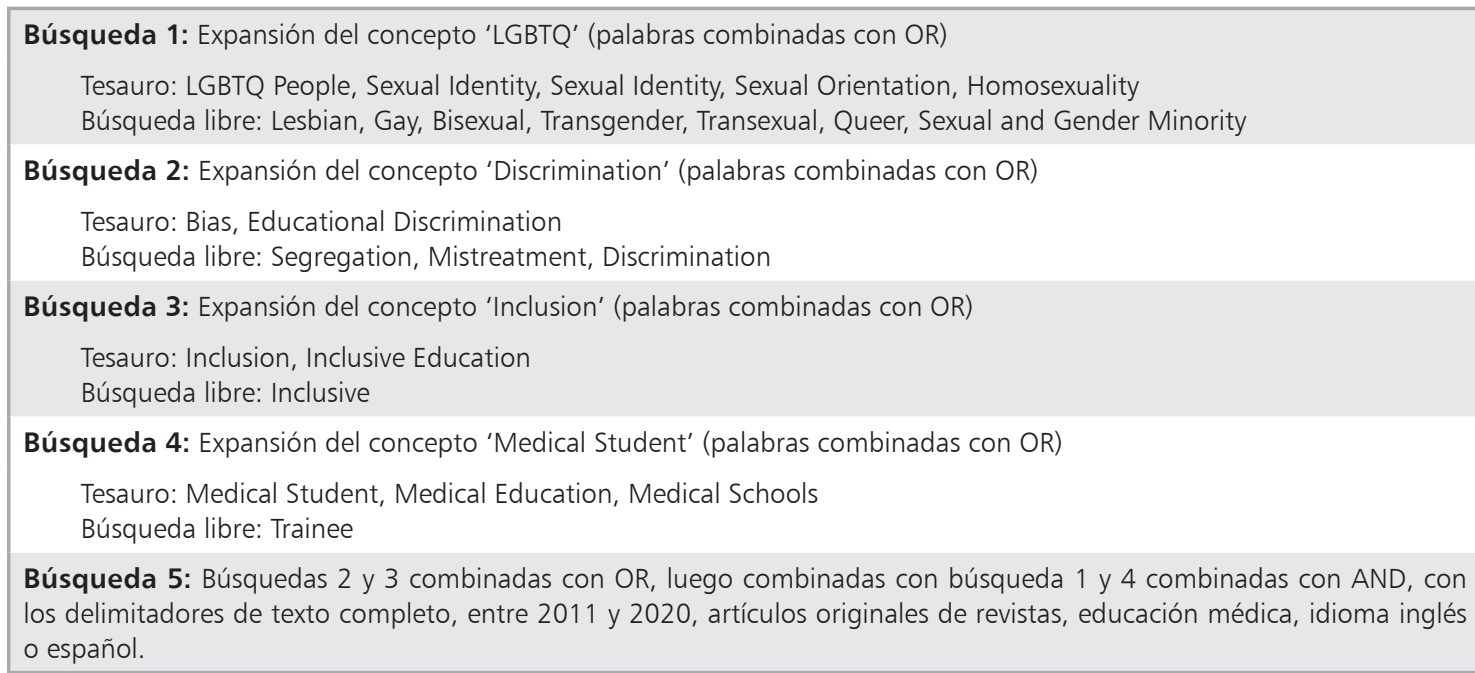

\section{Recopilación de datos}

Dos autores realizaron la extracción de la información de los artículos incluidos de forma independiente. Para esto, se revisó el texto completo, y se seleccionaron los datos que hicieran referencia a las experiencias de discriminación o inclusión de los estudiantes de medicina LGBTQ+. La discusión con otro autor resolvió cualquier diferencia en la extracción de datos. Los datos que hacían referencia a experiencias similares fueron agrupados bajo nombres comunes.

\section{Clasificación de las experiencias}

Posteriormente, las experiencias fueron clasificadas dentro de cuatro categorías emergentes: i) Relación entre pares en el contexto educativo; ii) Relación entre estudiantes y profesores en el contexto educativo; iii) Relación con la institución educativa y iv) Experiencia curricular y formativa. Además, se definió una experiencia significativa con un umbral de al menos $20 \%$ de apariciones en la literatura, las cuales fueron definidas.

\section{Resultados}

\section{Selección de los estudios}

La revisión sistemática identificó un total de 443 artículos. De estos, se excluyeron 57 artículos que se encontraban duplicados. De los 387 artícu- los restantes, 32 fueron seleccionados en función de su título y resumen. Se realizó una revisión de texto completo en los artículos seleccionados. Diecisiete estudios no cumplieron los criterios de inclusión. Finalmente, 15 estudios cumplieron con los criterios de inclusión.

\section{Características de los estudios}

Los estudios seleccionados se publicaron entre 1996 y 2020, con un aumento desde 2014, lo que refleja que el interés en la investigación en educación médica actual.

La representación de la población LGBTQ+ ha presentado variaciones en el tiempo. Los primeros estudios fueron publicados en 1996, y se encontraban focalizados principalmente en la población lesbiana, gay y bisexual. Posteriormente, se incluyó a la población transgénero y queer desde 2014. En 2019 , por primera vez, fueron publicados estudios focalizados exclusivamente en población transgénero y queer.

La mayoría de los artículos fueron conducidos en los Estados Unidos de Norteamérica (11/15). La metodología utilizada en estos estudios ha sido cualitativa, cuantitativa o mixta. Para recopilar la información, se utilizaron cuestionarios, entrevistas semiestructuradas e instrumentos específicos para evaluar la experiencia de los estudiantes. Se presenta un resumen de los artículos en la Tabla 2. 


\begin{tabular}{|c|c|c|c|c|c|}
\hline 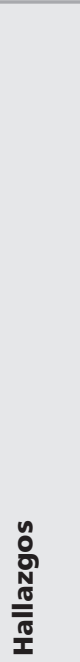 & 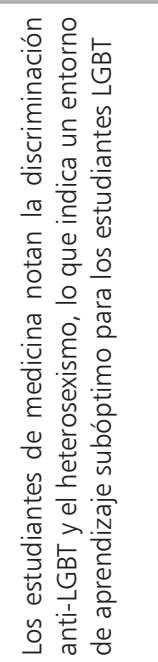 & 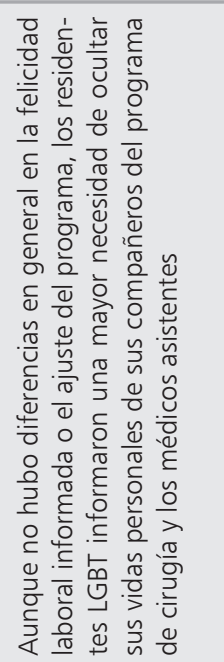 & 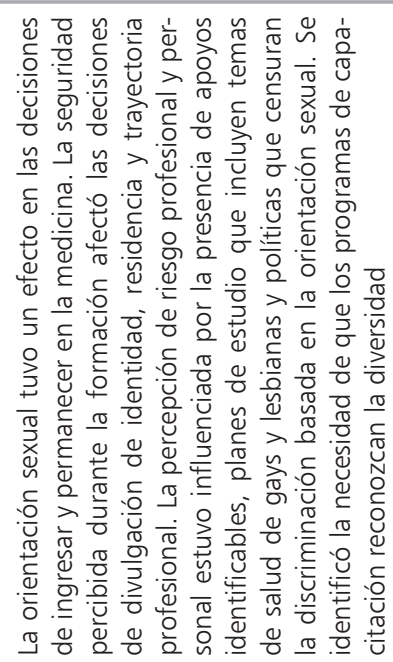 & 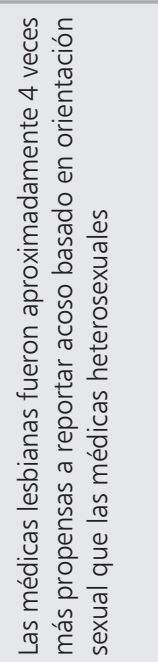 & 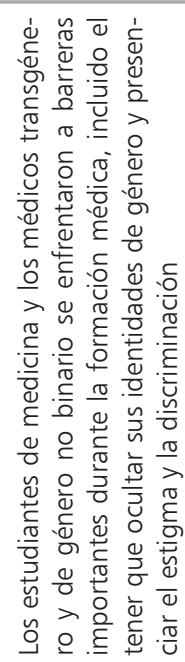 \\
\hline 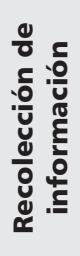 & 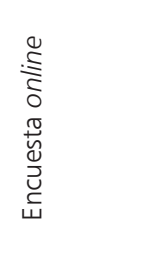 & 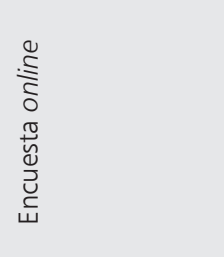 & 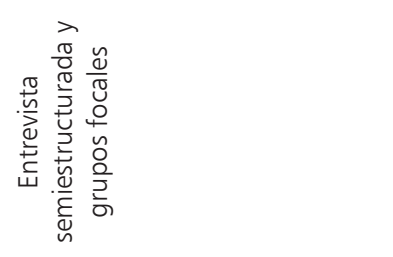 & 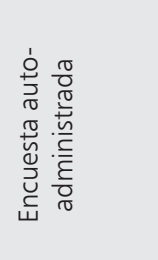 & 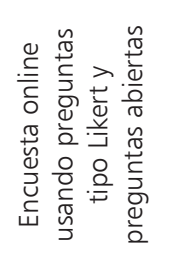 \\
\hline 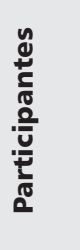 & 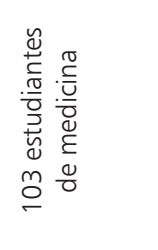 & 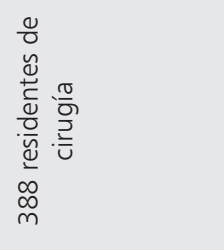 & 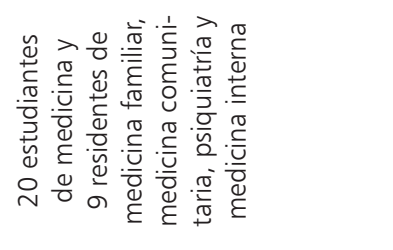 & 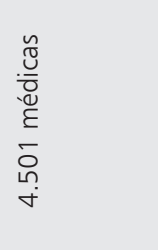 & 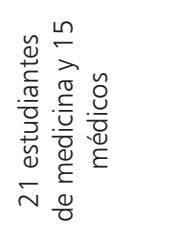 \\
\hline 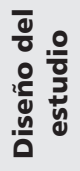 & 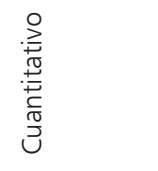 & 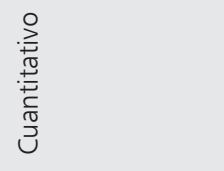 & 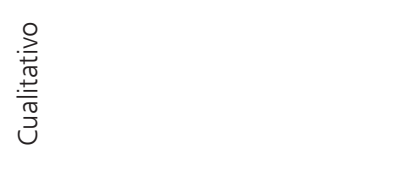 & 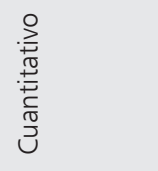 & 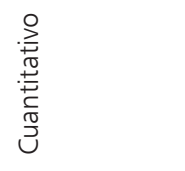 \\
\hline 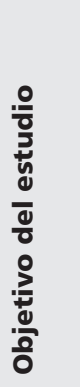 & 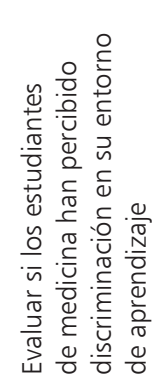 & 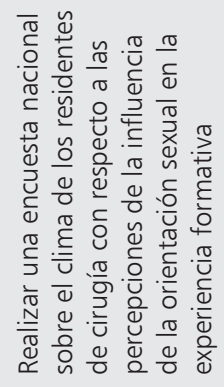 & 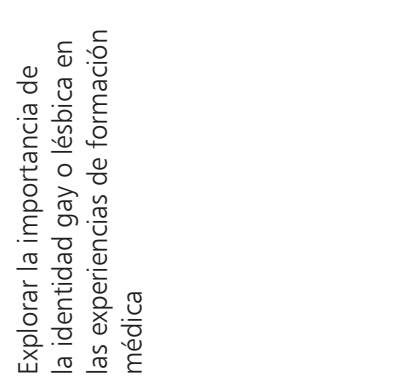 & 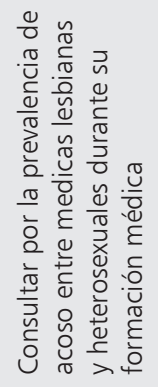 & 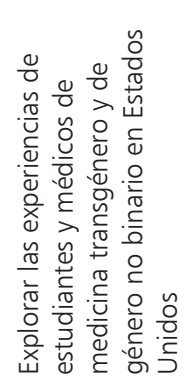 \\
\hline 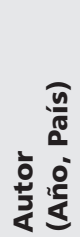 & 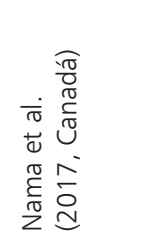 & 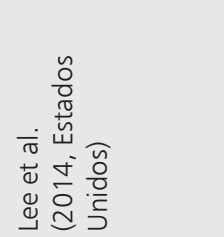 & 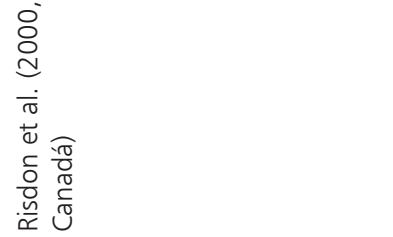 & 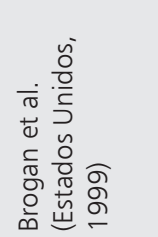 & 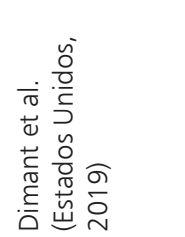 \\
\hline
\end{tabular}




\begin{tabular}{|c|c|c|c|c|}
\hline 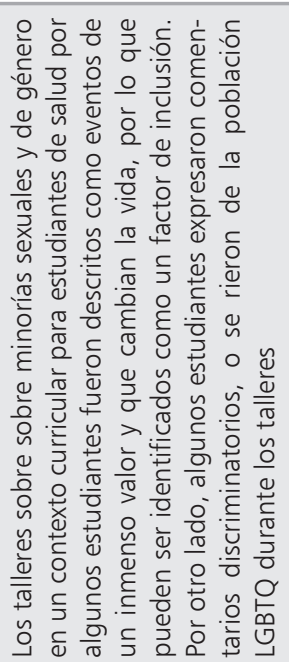 & 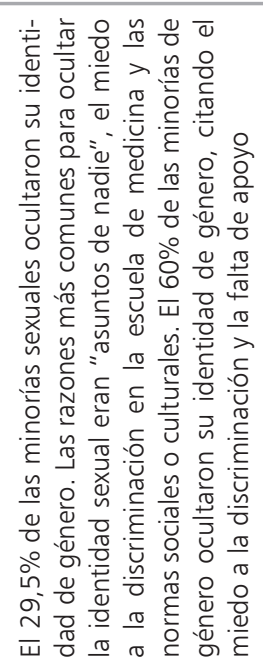 & 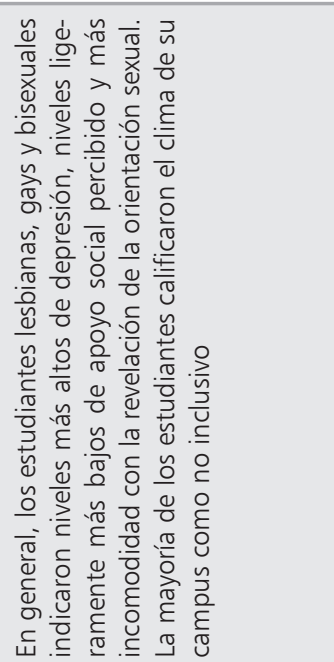 & 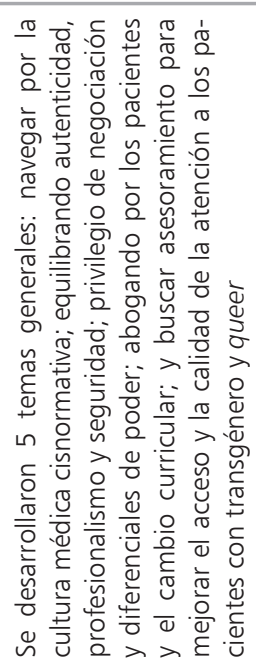 & 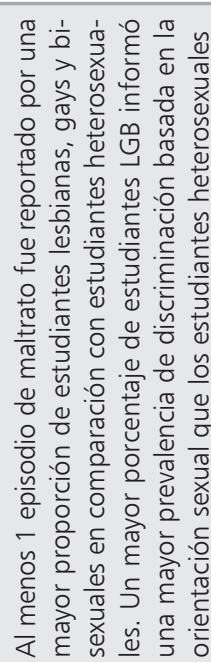 \\
\hline 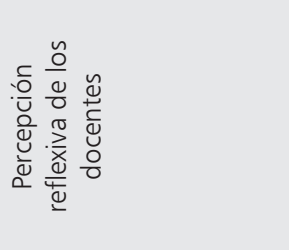 & 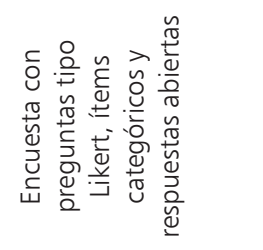 & 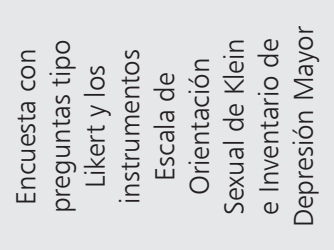 & 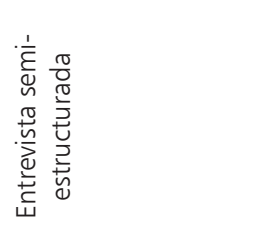 & 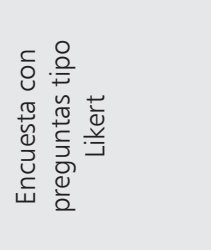 \\
\hline & 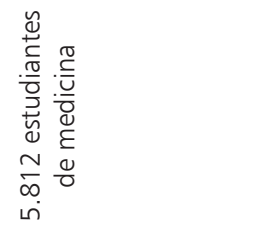 & 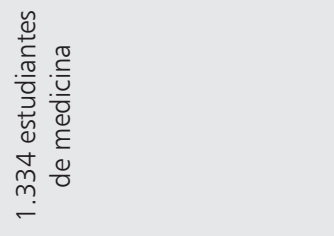 & 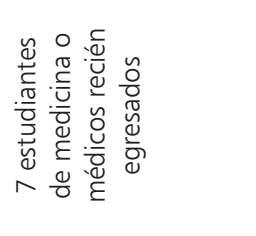 & 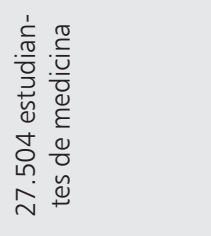 \\
\hline : & 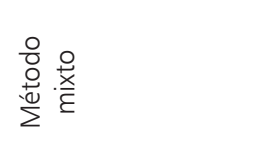 & 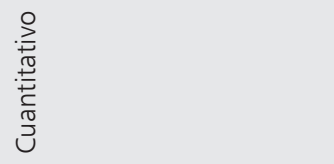 & 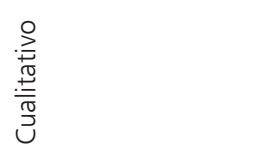 & 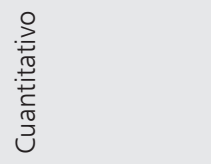 \\
\hline 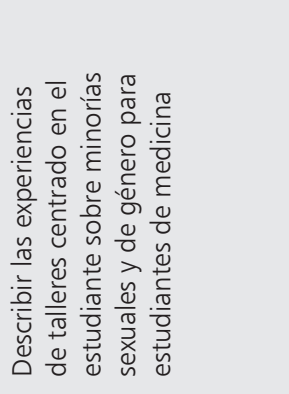 & 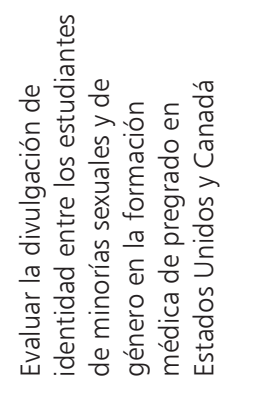 & 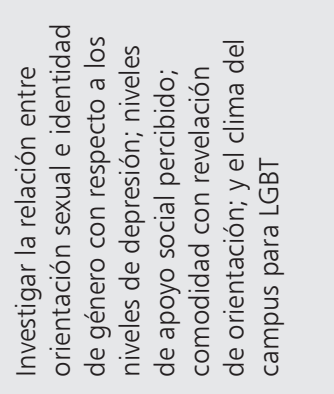 & 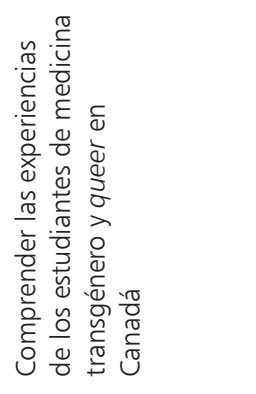 & 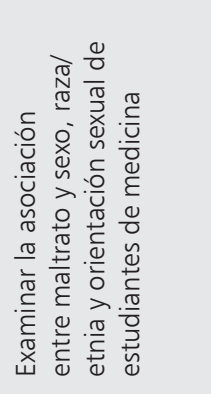 \\
\hline 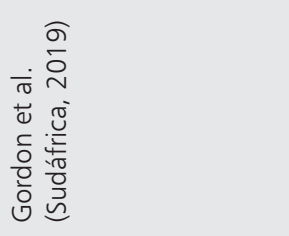 & 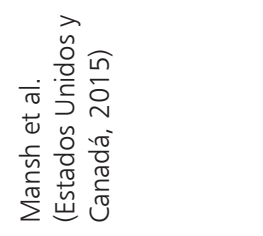 & 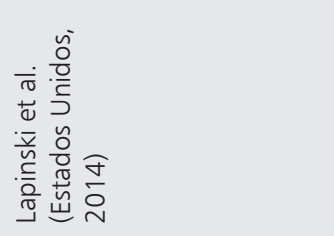 & 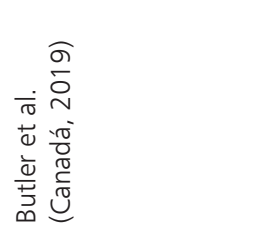 & 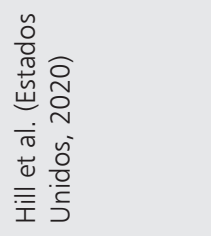 \\
\hline
\end{tabular}




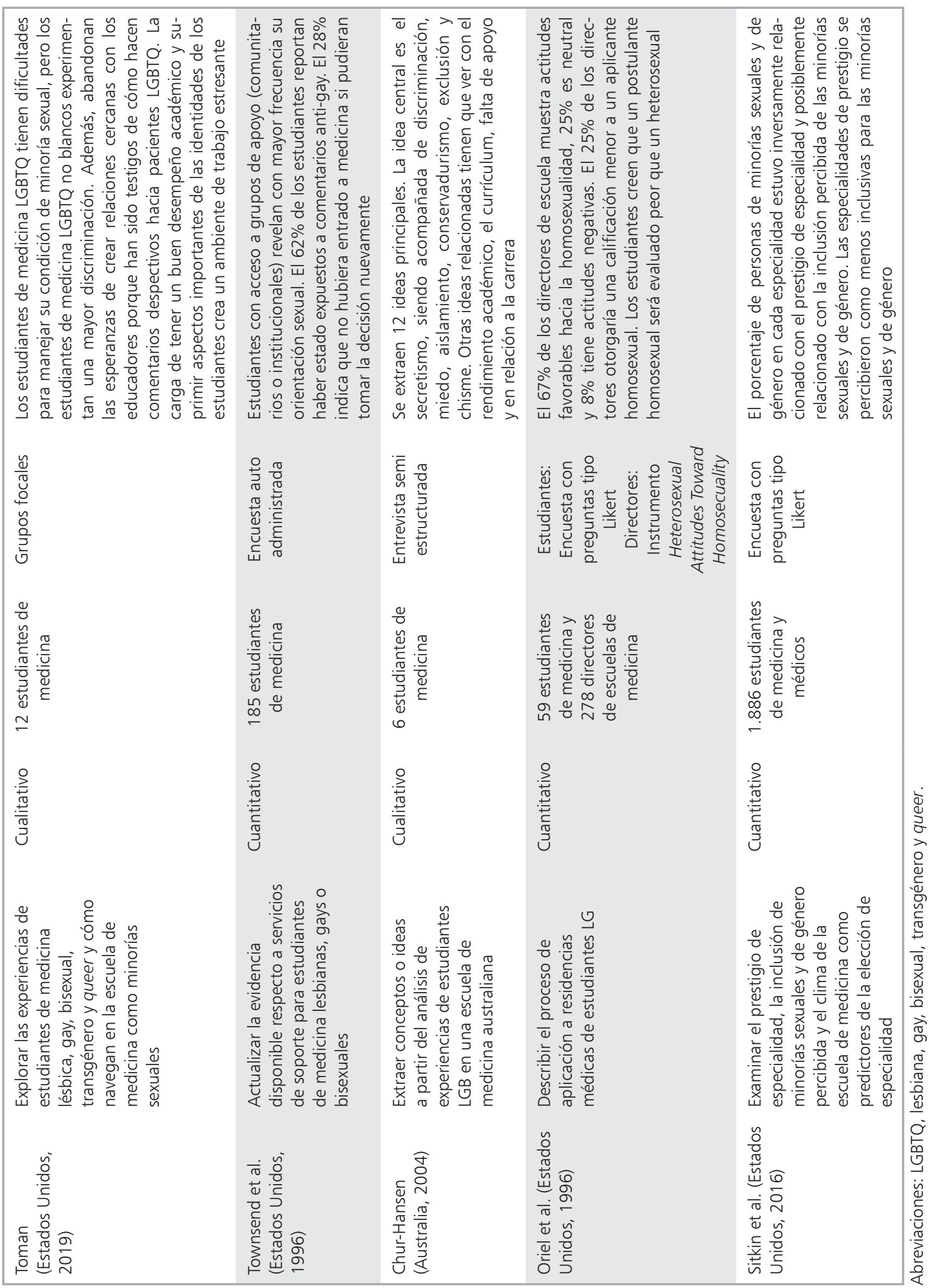




\section{Temas comunes encontrados en los estudios}

De los 15 estudios seleccionados, se identificaron 43 experiencias, que finalmente se clasificaron en 4 categorías: i) Relación entre pares en el contexto educativo; ii) Relación entre estudiantes y profesores en el contexto educativo; iii) Relación con la institución educativa y iv) Experiencia curricular y formativa. En términos del total de experiencias identificadas, obtuvieron un peso relativo de $23 \%$, 23\%, 34\% y $19 \%$, respectivamente. En cada una de ellas, se incluyeron todos los aspectos correspondientes mencionados en los artículos (Tabla 3).

\section{Relación entre pares en el contexto educativo}

Corresponde a las experiencias de los estudiantes de medicina LGBTQ+ al interactuar con sus compañeros dentro del ámbito académico. Estas fueron mencionadas en 11 de los 15 artículos $(73,3 \%)$.

La experiencia de escuchar comentarios discriminatorios hacia la población LGBTQ+ por parte de compañeros fue la experiencia más frecuentemente expuesta ${ }^{8-13}$, incluyendo comentarios dirigidos hacia otros compañeros, trabajadores de la salud y pacientes. La segunda experiencia más frecuente, con 4 menciones, fue sentir que la pertenencia social al grupo de compañeros era influenciada por la raza, el género y la orientación sexual $^{12,14-16}$, con la existencia de un sentimiento de aislamiento por parte de los estudiantes LGB$\mathrm{TQ}+$, que resultaba especialmente intenso en los estudiantes que además pertenecían a minorías étnicas y raciales ${ }^{9}$. Otra experiencia importante fue ocultar su identidad de género u orientación sexual por temor al rechazo ${ }^{8,9,12}$. Finalmente, como única experiencia positiva, se menciona el compartir con compañeros que dan apoyo a la comunidad LGBTQ+ ${ }^{12,14,16}$.

\section{Relación entre estudiantes y profesores en el contexto educativo}

Corresponde a las experiencias de los estudiantes de medicina LGBTQ+ al interactuar con sus profesores o tutores clínicos dentro del ámbito académico y formativo. Estas fueron mencionadas en 10 de los 15 artículos (66,6\%).

$\mathrm{Al}$ igual que en la relación entre pares, la relación entre estudiantes con sus profesores estaba marcada por escuchar comentarios discriminatorios hacia la población LGBTQ+ ${ }^{8,9,11-13,16,17}$, con 7 menciones. Los segundos aspectos más frecuentes, con 4 menciones, fueron el ocultar en forma activa la propia identidad de género u orientación sexual a sus profesores por temor al rechazo o repercusiones negativas $s^{8,12,15,18}$. Además, recibir personalmente comentarios discriminatorios basados en orientación sexual e identidad de género ${ }^{8,11,16,19}$.

Presenciar modelos de conducta discriminatorios en la atención de pacientes LGBTQ+ fue otra experiencia relevante, con 3 menciones ${ }^{12,16,17}$, incluyendo el observar a médicos hacer comentarios discriminatorios sobre pacientes ${ }^{15,17}$ y tener un trato inadecuado en la atención de estos ${ }^{17}$. Asimismo, se consideraron experiencias relevantes, con 3 menciones, el manifestar temor a poner en peligro oportunidades profesionales por pertenecer a la comunidad LGBTQ $+{ }^{12,16,20}$, y sentir que la pertenencia al grupo de compañeros era influenciada por raza, género y orientación sexual ${ }^{12,16,17}$.

\section{Relación con la institución educativa}

Tiene relación con las experiencias de los estudiantes de medicina LGBTQ+ al interactuar con las escuelas de medicina y las estructuras formales de las instituciones de educación superior, incluyendo autoridades o estamentos oficiales. Estas fueron mencionadas en 10 de los 15 artículos $(66,6 \%)$.

El temor a la discriminación en los procesos de admisión por parte de las instituciones hacia postulantes de la comunidad LGBTQ+ fue la experiencia más frecuentemente expuesta en esta categoría, con 4 menciones ${ }^{8,11,13,17}$. En este proceso, el postulante enfrenta una entrevista personal con autoridades institucionales.

El reconocer la existencia de políticas que censuran explícitamente la discriminación basada en orientación sexual fue la segunda experiencia más frecuente, con 3 menciones ${ }^{9,14,21}$.

\section{Experiencia curricular y formativa}

Se refiere a aquellas experiencias que tienen relación con el proceso formativo y, en particular, con aspectos relacionados con el currículum de la carrera. Estas fueron mencionadas en 10 de los 15 artículos $(66,6 \%)$.

Reconocer la influencia de la propia orientación sexual o identidad de género en la elección de ser médico o de especialidad fue la experiencia más frecuente, con 5 menciones ${ }^{8,9,12,13,22}$. Por ejemplo, se señala que la decisión de estudiar medicina fue 
Tabla 3. Clasificación de las experiencias de discriminación o inclusión de los estudiantes de medicina pertenecientes a la diversidad sexual y de género

\section{Relación entre pares en el contexto educativo}

- Escuchar comentarios discriminatorios hacia la población LGBTQ (6)

- Sentir pertenencia social al grupo de compañeros influenciado por raza, género y orientación sexual (4)

- Compartir con compañeros que dan apoyo a comunidad LGBTQ (3)*

- Ocultar identidad de género u orientación sexual por temor al rechazo (3)

- Leer comentarios transfóbicos en grupos de compañeros en redes sociales (2)

- Manifestar sentimiento de no pertenencia a grupo de compañeros (2)

- Presenciar la atención discriminatoria de pacientes LGBTQ (2)

- Recibir comentarios discriminatorios basados en DSG (2)

- Tener pares que pertenecen a grupos conservadores, religiosos u homofóbicos (2)

- Sentir temor a ser discriminado por identidad de género u orientación sexual (1)

II. Relación entre estudiantes y profesores en el contexto educativo

- Escuchar comentarios discriminatorios hacia la población LGBTQ (7)

- Ocultar identidad de género u orientación sexual por temor al rechazo (4)

- Recibir comentarios discriminatorios basados en DSG (4)

- Manifestar temor a poner en peligro oportunidades profesionales por pertenecer a comunidad LGBTQ (3)

- Presenciar modelos de conducta discriminatorios en la atención de pacientes LGBTQ (3)

- Sentir pertenencia social en la interacción con profesores influenciado por raza, género y orientación sexual (3)

- Presenciar la inclusión de contenido discriminatorio hacia la población LGBT (2)

- Sentir temor a recibir evaluaciones sesgadas por pertenecer a DSG (2)

- Sentir temor a ser discriminado por identidad de género u orientación sexual (2)

- Escuchar uso de lenguaje inclusivo por parte de profesores (1)*

III. Relación con la institución educativa

- Temor a discriminación en procesos de admisión (4)

- Reconocer la existencia de políticas que censuran explícitamente discriminación basada en orientación sexual e identidad de género (3)*

- Confiar y conocer sobre políticas antidiscriminación (2)*

- Contar con servicios específicos para estudiantes LGBTQ (2)*

- Reconocer la creación y promoción de espacios seguros $(2)^{*}$

- Temor a una institución conservadora o religiosa (2)

- Temor a perder oportunidades de entrenamiento o recompensas debido a la orientación sexual (1)

- Identificar la ausencia de miembros abiertamente LGBTQ en la institución (1)

- Identificar la necesidad de políticas de cambio de nombre para estudiantes transgénero (1)*

- Manifestar la necesidad de organizaciones para estudiantes LGBTQ (1)

- Observar una inapropiada asignación de espacios según género (e.g., baños, casilleros) (1)

- Plantear la presunción de un estudiantado heterosexual (1)

- Reconocer la Inclusión de DSG en declaraciones públicas (1)

- Reconocer la presencia de apoyo por parte de la Escuela de Medicina (1)

- Temor a ser discriminado por parte de la administración (1)

\section{Experiencia curricular y formativa}

- Reconocer la influencia de la propia orientación sexual o identidad de género en la elección de ser médico o de seguir una especialidad específica (5)

- Identificar la inclusión de temas de salud LGBTQ en el currículum (3)*

- Percibir una cobertura curricular insuficiente sobre temas de salud LGBTQ (3)

- Identificar supuestos heterosexistas en el plan de estudios (2)

- Constatar la presencia de talleres de DSG en contexto curricular (1)*

- Identificar contenidos transfóbicos en las clases (1)

- Integrar contenidos LGBTQ en cursos existentes (1)

- Percibir que los cambios curriculares no tratan transversalmente la temática (1)

La frecuencia en que cada experiencia fue mencionada en los diferentes artículos se encuentra entre paréntesis al lado de cada experiencia. Las experiencias positivas fueron señaladas con asterisco. Abreviaciones: DSG, diversidad sexual y de género; LGBTQ, lesbiana, gay, bisexual, transgénero y queer. 
Tabla 4. Recomendaciones para la inclusión de los estudiantes de medicina de la diversidad sexual y de género

A nivel institucional

- Se requiere generar políticas preventivas y de gestión de casos de discriminación basada en DSG

- Considerar la diversidad como un valor a promover y reforzar en todos sus niveles y dimensiones

- Desarrollar políticas flexibles para el cambio de nombre en personas transgénero que se encuentran realizando su transición

- Formar en la comunidad universitaria (docentes, funcionarios, estudiantes) en la alfabetización actual en el enfoque de DSG

- Las escuelas de medicina deberían implementar o ampliar espacios de participación e integración de la diversidad, de cualquier tipo

- A nivel de los espacios educativos, considerar baños de un solo cubículo como disponibles para personas de todos los géneros

\section{A nivel curricular}

- Integrar formalmente aprendizajes y contenidos relacionados a salud de personas LGBTQ + de forma transversal en los cursos existentes

- Considerar experiencia práctica con pacientes LGBTQ + en ambientes simulados o en atención clínica con la tutela de un profesional capacitado

A nivel de interacciones interpersonales

- Promover grupos de apoyo patrocinados por la institución que reconozcan y permitan la discusión de la experiencia de pertenecer a la DSG durante la formación médica

- Promover lenguaje inclusivo, evitando comentarios discriminatorios por parte de tutores o estudiantes hacia la población LGBTQ+

Abreviaciones: DSG, diversidad sexual y de género; LGBTQ, lesbiana, gay, bisexual, transgénero y queer.

tomada en parte para "compensar" lo negativo de su orientación sexual". Además, se plantea una menor probabilidad de elegir programas de especialidad considerados menos inclusivos con la población LGBTQ+ y que no cuenten con miembros abiertamente LGBTQ+.

Se identificaron 2 experiencias relevantes relacionadas a contenidos curriculares sobre la población LGBTQ+, presentando 3 menciones cada una. Por un lado, el identificar la inclusión de temas de salud de la población LGBTQ+ en las mallas curriculares de las escuelas de medicina fue referido como una experiencia positiva ${ }^{8,9,18}$. Por otro lado, como una experiencia negativa, se percibe una insuficiente cobertura curricular sobre temas de salud de la población LGBTQ $+{ }^{12,14,16}$, conceptualizada como la ausencia de contenidos relevantes ${ }^{15}$, el escaso tiempo destinado ${ }^{12,14}$ y la falta de instancias con pacientes LGBTQ+ (casos clínicos o atención de pacientes) ${ }^{12,14}$.

\section{Discusión}

El número de experiencias identificadas para cada categoría fue heterogéneo, destacando la rela- ción con la institución educativa como la categoría más mencionada. La mayoría de las experiencias reportadas correspondían a experiencias negativas durante la formación médica.

La relación entre pares en el contexto educativo fue mencionada en $73,3 \%$ de los artículos. Los estudiantes que tienen problemas con las relaciones con sus compañeros en la universidad pueden tener dificultades para adaptarse, incluso el aislamiento y la escasa integración social se han identificado como razones comunes de deserción ${ }^{23}$. Promover las relaciones sociales entre pares puede disminuir la angustia y bajo rendimiento académico ${ }^{24}$.

La relación entre estudiantes y profesores en el contexto educativo fue una categoría mencionada en $66,6 \%$ de los artículos, resultando mencionadas fundamentalmente experiencias negativas. Estas relaciones interpersonales tienen efectos en ambas partes. Por un lado, los profesores que mantienen relaciones interpersonales saludables con sus alumnos experimentan mayor satisfacción laboral y menor agotamiento ${ }^{25}$. Por otro lado, las percepciones de los estudiantes sobre la interacción con sus profesores están asociadas con la motivación, el desempeño y la satisfacción con el curso ${ }^{25,26}$. La 
relación profesor-estudiante requiere habilidades interpersonales efectivas para lograr resultados satisfactorios $^{27}$, las cuales son especialmente relevantes en la interacción con los estudiantes LGBTQ+, quienes experimentan discriminación al interactuar con sus profesores.

La relación con la institución educativa fue mencionada en $66,6 \%$ de los artículos. Esta relación se ha conceptualizado como la interacción entre estudiantes y su universidad, incluyendo aspectos como la reciprocidad, la confianza, el compromiso, la satisfacción y los sentimientos de los estudiantes sobre su institución ${ }^{28}$. Es una interacción relevante, puesto que se ha reportado que los estudiantes con un fuerte sentido de pertenencia a su institución tienen más probabilidades de ser persistentes y esforzarse en el aprendizaje $\mathrm{e}^{28}$, mientras que los estudiantes que no se sienten conectados con la institución corren el riesgo de abandonar los estudios ${ }^{23}$.

La experiencia curricular y formativa de los estudiantes de medicina LGBTQ+ fue mencionada en $66,6 \%$ de los artículos. En consideración con la importancia del currículum, resulta necesaria la generación de políticas institucionales para resguardar un ambiente educativo favorecedor del desarrollo académico ${ }^{29}$. En este contexto, ha sido descrito que cuando los estudiantes pertenecientes a minorías se enfrentan con un plan de estudios que es insensible a sus diferencias, estos pueden tener un desempeño inadecuado ${ }^{30}$. Por otra parte, los planes de estudio que expresan apoyo a la población LGBTQ+ se han asociado con una mayor percepción de seguridad y menores niveles de maltrato basado en DSG ${ }^{31}$. Es por esto que es necesario el apoyo de las instituciones para implementar cambios en el currículum ${ }^{32}$.

\section{Fortalezas y limitaciones}

Esta revisión sistemática es, hasta el conocimiento de los autores, la primera que sintetiza las experiencias de los estudiantes de medicina LGBTQ+ durante su formación.

Este estudio presenta varias limitaciones. La mayoría de los estudios fueron conducidos en Norteamérica, lo que podría afectar la validez externa. Además, debido a la heterogeneidad de los estudios, no fue posible realizar un metaanálisis. Finalmente, no fue posible el registro del protocolo de investigación utilizado en una base de datos pública.

\section{Investigaciones futuras}

Los individuos de la DSG, particularmente aquellos con identidades estigmatizadas coexistentes, experimentan una carga desproporcionada de discriminación social ${ }^{12,14-17}$, por lo que sería recomendable que futuras investigaciones aborden este problema desde la interseccionalidad ${ }^{33}$. Además, se deben evaluar estrategias para mejorar la inclusión de la DSG en las escuelas de medicina, y para abordar las deficiencias en los planes de estudios.

\section{Recomendaciones}

Nuestro estudio puede ser sintetizado en recomendaciones a tres niveles, en vista a transitar desde discriminación hacia la inclusión de los estudiantes de medicina LGBTQ+ durante su formación (Tabla 4).

\section{Conclusión}

A pesar de los avances generados en las últimas décadas, los estudiantes de medicina pertenecientes a la población LGBTQ+ aún experimentan discriminación durante su formación. Es necesario que las escuelas de medicina generen estrategias que permitan transformar la educación médica en un espacio más inclusivo para la población LGBTQ+, tanto por el bienestar de los propios estudiantes y en la construcción de una sociedad tolerante y diversa; como para la formación de profesionales sensibles a las necesidades de una población que sufre inequidades en salud.

\section{Referencias}

1. Wittlin N, Dovidio J, Burke S. Contact and Role Modeling Predict Bias Against Lesbian and Gay Individuals Among Early-Career Physicians: A Longitudinal Study. Soc Sci Med. 2019; 238: 112422. doi:10.1016/j.socscimed.2019.112422.

2. Sorensen J, Norredam M, Dogra N, Essink-Bot ML, Suurmond J, Krasnik A. Enhancing cultural competence in medical education. Int J Med Educ. 2017; 8: 28-30. doi:10.5116/ijme.587a.0333.

3. Lanting K, Dogra N, Hendrickx K, Sim J, Suurmond J. Culturally Competent in Medical Education-European Medical Teachers' Self-Reported Preparedness and Training Needs to Teach Cultural Competence Topics and to Teach a Diverse Class. MedEdPublish. 2019; 8 (2): 28. 
4. Sanlo R. Lesbian, Gay, and Bisexual College Students: Risk, Resiliency, and Retention. J Coll Student Retent. 2004; 6 (1): 97-110. doi:10.2190/fh61-ve7v-hhcx0pur.

5. Lange AC, Durán A, Jackson R, Lange AC, Durán A, Jackson R. The State of LGBT and Queer Research in Higher Education Revisited: Current Academic Houses and Future Possibilities. J Coll Stud Dev. 2019; 60 (5): 511-26. doi:https://doi.org/10.1353/csd.2019.0047.

6. Ellis SJ. Diversity and inclusivity at university: a survey of the experiences of lesbian, gay, bisexual and trans (LGBT) students in the UK. High Educ. 2008; 57 (6): 723-39. doi:10.1007/s10734-008-9172-y.

7. Liberati A, Altman DG, Tetzlaff J, Mulrow C, Gøtzsche PC, John PA. The PRISMA statement for reporting systematic reviews and meta-analyses of studies that evaluate healthcare interventions explanation and elaboration. BMJ. 2009; 339. doi:10.1136/bmj.b2700.

8. Lee KP, Kelz RR, Dubé B, Morris JB. Attitude and Perceptions of the Other Underrepresented Minority in Surgery. J Surg Educ. 2014; 71 (6): e47-e52. doi: 10.1016/j.jsurg.2014.05.008.

9. Risdon C, Cook D, Willms D. Gay and lesbian physicians in training: a qualitative study. Can Med Assoc J. 2000; 162 (3): 331-4.

10. Gordon C, Mitchell V. Risks and Rewards in Sexual and Gender Minority Teaching and Learning in a South African Health Sciences Medical Curriculum. Educ as Chang. 2019; 23 (3757). doi: https://doi. org/10.25159/1947-9417/3757.

11. Mansh M, White W, Gee-Tong L. Sexual and Gender Minority Identity Disclosure During Undergraduate Medical Education: "In the Closet" in Medical School. Acad Med. 2015; 90 (5): 634-44. doi: 10.1097/ ACM.0000000000000657.

12. Chur-Hansen A. Experience of being gay, lesbian or bisexual at an Australian medical school: a qualitative study. Int J Incl Educ. 2004; 8 (3): 281-91. doi: 10.1080/1360311032000160607.

13. Oriel KA, Madlon-Kay DJ, Govaker D, Mesy DJ. Gay and Lesbian Physicians in Traingin Family Practice Program Directors' Attitudes and Students' Perceptions of Bias. Fam Med. 1996; 28 (10): 720-5.

14. Butler K, Yak A, Veltman A. "Progress in Medicine Is Slower to Happen": Qualitative Insights Into How Trans and Gender Nonconforming Medical Students Navigate Cisnormative Medical Cultures at Canadian Training Programs. Acad Med. 2019; 94 (11): 17571765. doi: 10.1097/ACM.0000000000002933.

15. Townsend MH, Wallick MM, Cambre KM. Follow-up survey of support services for lesbian, gay, and bisexual medical students. Acad Med. 1996; 71 (9): 1012-4. doi: 10.1097/00001888-199609000-00018.

16. Toman L. Navigating medical culture and LGBTQ identity. Clin Teach. 2019; 16 (4): 335-8. doi:10.1111/ tct.13078.

17. Dimant OE, Cook TE, Greene RE, Radix AE. Health Experiences of Transgender and Gender Nonbinary Medical Students and Physicians. Transgender Heal. 2019; 4 (1): 209-17. doi: 10.1089/trgh.2019.0021.

18. Nama N, MacPherson P, Sampson M, McMillan HJ. Medical students' perception of lesbian, gay, bisexual, and transgender (LGBT) discrimination in their learning environment and their self-reported comfort level for caring for LGBT patients: a survey study. Med Educ Online. 2017; 22 (1): 1368850. doi: 10.1080/10872981.2 017.1368850 .

19. Brogan D, Frank E, Elon L, Sivanesan P. Harassment of Lesbians as Medical Students and Physicians. JAMA. 1999;282(13):1290-1292. doi: 10.1001/jama.282.13.1290-JMS1006-5-1.

20. Hill KA, Samuels EA, Gross CP. Assessment of the Prevalence of Medical Student Mistreatment by Sex, Race/Ethnicity, and Sexual Orientation. JAMA Intern Med. 2020; 180 (5): 653-65. doi: 10.1001/jamainternmed.2020.0030.

21. Lapinski J, Sexton P. Still in the closet: the invisible minority in medical education. BMC Med Educ. 2014; 14 (171). doi: 10.1186/1472-6920-14-171.

22. Sitkin NA, Pachankis JE. Specialty Choice Among Sexual and Gender Minorities in Medicine: The Role of Specialty Prestige, Perceived Inclusion, and Medical School Climate. LGBT Heal. 2016; 3 (6): 451-60. doi: 10.1089/ lgbt.2016.0058.

23. Maunder RE. Students' peer relationships and their contribution to university adjustment: the need to belong in the university community. J Furth High Educ. 2017; 42 (6): 756-68. doi: 10.1080/0309877X.2017.1311996.

24. Yamada Y, Klugar M, Ivanova K, Oborna I. Psychological distress and academic self-perception among international medical students: the role of peer social support. BMC Med Educ. 2014; 14 (1): 256. doi: 10.1186/ s12909-014-0256-3.

25. Maulana R, Opdenakker M, Brok P Den. Teacher-student interpersonal relationships in Indonesia: profiles and importance to student motivation. Asia Pacific J Educ. 2011; 31 (31): 33-49. doi: 10.1080/02188791.201 1.544061 .

26. Hagenauer G, Volet SE. Teacher-student relationship at university: an important yet under-researched field. Oxford Rev Educ. 2014; 40 (3): 370-88. doi: 10.1080/03 054985.2014.921613. 
27. Frymier AB, Houser ML. The teacher-student relationship as an interpersonal relationship The Teacher-Student Relationship as an Interpersonal Relationship. Commun Educ. 2000; 49 (3): 207-19.

28. Sung M, Yang S. Student-university relationships and reputation: a study of the links between key factors fostering students' supportive behavioral intentions towards their university. High Educ. 2009; 57 (6): 787 811. doi: 10.1007/s10734-008-9176-7.

29. Ortega J, Fasce E, Pérez C, Ibáñez P, Márquez C, Parra P. Evaluación de componentes del currículum oculto en estudiantes de medicina. Rev Med Chile 2014; 142 (11): 1452-7. doi: http://dx.doi.org/10.4067/S003498872014001100013.

30. Yuan H. Respond to Diversity: Graduate Minority Stu- dents' Perceptions on Their Learning Experiences in an American University. Int Acad Forum J Educ. 2017; 5 (1): 33-45. doi: https://doi.org/10.22492/ije.5.1.02.

31. Snapp SD, McGuire JK, Sinclair KO, Gabrion K, Russell ST. LGBTQ-inclusive curricula: why supportive curricula matter. Sex Educ. 2015; 15 (6): 580-96. doi: 10.108 0/14681811.2015.1042573.

32. Valenzuela-Valenzuela AV, Cartes-Velásquez R. Implicaciones en pacientes mujeres y LGBT+, estudiantes y profesores. Iatreia. 2020; 33 (1): 59-67. doi: 10.17533/ udea.iatreia.32.59.

33. Davis K, Davis K. Intersectionality as buzzword: A sociology of science perspective on what makes a feminist theory successful. Fem Theory. 2008; 9 (1): 67-85. doi: $10.1177 / 1464700108086364$. 